\title{
Dual antiplatelet therapy versus aspirin monotherapy in diabetics with stable ischemic heart disease undergoing coronary artery bypass grafting
}

\author{
Makoto Mori ${ }^{1}$, Kayoko Shioda ${ }^{2}$, Syed Usman Bin Mahmood ${ }^{1}$, Abeel A. Mangi ${ }^{1}$, James J. Yun ${ }^{1}$, Arnar \\ Geirsson $^{1}$
}

${ }^{1}$ Section of Cardiac Surgery, Yale University School of Medicine, New Haven, CT, USA; ${ }^{2}$ Department of Epidemiology of Microbial Diseases, Yale School of Public Health, New Haven, CT, USA

Correspondence to: Arnar Geirsson, MD, FACS, FAHA. Associate Professor of Surgery, Section of Cardiac Surgery, Yale School of Medicine, BB204, 330 Cedar Street, New Haven, CT 208039, USA. Email: arnar.geirsson@yale.edu.

\begin{abstract}
Background: Dual antiplatelet therapy (DAPT) in patients presenting with acute coronary syndrome (ACS) undergoing CABG is recommended to prevent recurrent ischemic events. The benefit of DAPT post-CABG in patients with stable ischemic heart disease (SIHD) is unknown. The aim of this study was to evaluate the utilization rate of DAPT and associated outcomes in patients with SIHD undergoing CABG via a secondary analysis of Bypass Angioplasty Revascularization Investigation 2 Diabetes (BARI 2D) trial data.

Methods: In a post-hoc, nonrandomized analysis from the BARI 2D trial, we compared patients receiving DAPT and aspirin monotherapy within 90 days post-randomization. The primary outcome was the risk adjusted 5-year composite of all-cause mortality, nonfatal myocardial infarction (MI), or stroke. We analyzed patients assigned to prompt CABG treatment arm including both the insulin therapy assignments.

Results: Of 378 patients, within 90 days post-randomization, 59 (16\%) patients received DAPT and $319(84 \%)$ patients received aspirin alone. Cox proportional hazard analysis demonstrated that there was no significant difference in the 5-year composite event of death, MI, and stroke between DAPT and monotherapy cohorts [13 (22.0\%) vs. 61 (19.1\%); adjusted hazard ratio (HR): 1.06; 95\% confidence interval (CI): 0.56 to $2.00 ; \mathrm{P}=0.86$. There also was no significant difference at 1 year in the composite event [6 (10.2\%) vs. 30 (9.4\%); HR: 1.13 ; 95\% CI: 0.46 to 2.79 ; $\mathrm{P}=0.79$ ].

Conclusions: The use of DAPT in patients with diabetes post-CABG in this cohort was low. Compared with aspirin monotherapy, no associated differences were observed in cardiovascular outcomes. Larger prospective studies are needed to further elucidate this observation.
\end{abstract}

Keywords: Dual antiplatelet therapy (DAPT); coronary artery bypass grafting (CABG); Bypass Angioplasty Revascularization Investigation 2 Diabetes (BARI 2D); stable ischemic heart disease (SIHD)

Submitted Mar 26, 2018. Accepted for publication Aug 02, 2018.

doi: 10.21037/acs.2018.08.01

View this article at: http://dx.doi.org/10.21037/acs.2018.08.01

\section{Background}

Resumption of DAPT post-CABG in patients presenting with acute coronary syndrome (ACS) is supported by national (1) and international guidelines (2,3), although there remains lack of robust evidence supporting its use. This recommendation focusing on ACS, without inclusion of SIHD, may be partly attributed to the historical sequence of trials that initially demonstrated benefits of DAPT use in patients presenting with ACS with or without further percutaneous coronary intervention (PCI) $(4,5)$. These findings were followed by the subgroup analysis of the CABG cohort in the Clopidogrel in Unstable angina to prevent Recurrent ischemic Events (CURE) trial (6), that demonstrated a trend toward reduced risk of adverse 
events in patients receiving DAPT compared to aspirin monotherapy.

Several trials have demonstrated the benefit of DAPT use in patients undergoing CABG in preserving vein graft patency $(7,8)$. These informative trials, however, included patients presenting with both ACS and with SIHD. Whether the presenting symptoms would translate into clinical differences relevant to antiplatelet therapy following CABG remain unanswered.

Bypass Angioplasty Revascularization Investigation 2 Diabetes (BARI 2D) Study Group conducted a randomized trial of therapies for patients with type 2 diabetes and SIHD (9), in which the optimal treatment for patients with type 2 diabetes (DM) and SIHD was investigated. The entire cohort was pre-assigned to CABG or PCI arm. The CABG arm of this trial data presents a unique opportunity to evaluate the potential utility of DAPT in patients with $\mathrm{DM}$ and SIHD who underwent CABG.

The aim of this secondary analysis is to evaluate whether, in the BARI 2D cohort, the use of DAPT is associated with the hazard of adverse events in patients with SIHD and DM undergoing CABG. We hypothesize that DAPT does not provide therapeutic advantage over aspirin monotherapy in patients presenting with SIHD who subsequently undergo CABG.

\section{Methods}

\section{Data source}

The method and results of the BARI 2D trial have been published (9). This multicenter international trial enrolled patients with type 2 diabetes and coronary artery disease by angiography ( $\geq 50 \%$ stenosis of a major epicardial coronary artery associated with a positive stress test or $\geq 70 \%$ stenosis of a major epicardial coronary artery and classic angina). Patients undergoing revascularization within 12 months prior to randomization were excluded. The trial utilized 2 by 2 factorial design, in which patients with coronary artery disease and diabetes were first randomly assigned to undergo either prompt coronary revascularization or medical therapy. Subsequently, patients were randomly assigned to undergo either insulinsensitization or insulinprovision therapy. Based on clinical discretion, the entire cohort was pre-assigned to $\mathrm{CABG}$ or PCI, creating eight treatment arms, each with a unique combination of revascularization strategy, medical therapy versus prompt revascularization, and insulin sensitization versus insulin provision.
Patients who received DAPT within 90-day postrandomization were defined as those receiving DAPT. The type of second antiplatelet agent could not be delineated from the trial data. Provided that the enrolment took place prior to the approval of novel P2Y12 inhibitors (prasugrel and ticagrelor), it is presumed that the second agents were neither of those medications.

\section{Outcomes}

The primary outcome was BARI 2D primary endpoint of 5 -year major adverse cardiac and cerebrovascular events (MACCE): all-cause mortality, non-fatal myocardial infarction (MI), or stroke. Secondary endpoints were defined as an individual component of the composite outcome, need for subsequent revascularization, and composite and individual outcomes at 1-year post-randomization. All events were adjudicated by a clinical event committee.

\section{Statistical methods}

Differences in the patient characteristics were compared with the two-tailed $t$-test, chi-square test, or Fisher's exact test, where appropriate. Continuous variables are expressed in mean with standard deviation (SE) format unless otherwise specified. The time to primary and secondary endpoints were assessed with Cox proportional hazard regression. The following variables were included in the model as potential confounders: age, insulin treatment arm assignment, history of MI, myocardial jeopardy score, prior coronary stent placement, prior CABG, history of stroke, number of totally occluded lesions, number of lesions with $\geq 70 \%$ stenosis, number of lesions with $\geq 50 \%$ stenosis, and $\geq 50 \%$ stenosis in proximal left anterior descending artery. Variables were removed from the model when they did not satisfy the proportionality assumption. A $\mathrm{P}$ value of $<0.05$ was used to define statistically significant difference and correlations. All analysis was conducted with SAS 9.4 (SAS Institute Inc., Cary, NC, USA) and R version 3.4.2 (Vienne, Austria). The Yale Institutional Review Board and the National Heart, Lung, and Blood Institute (NHLBI) Biologic Specimen and Data Repository Information Coordinating Center approved this study (protocol ID: 2000020935).

\section{Results}

The entire participant cohort of BARI 2D trial consisted 
of 2,368 patients. Within this cohort, 763 patients were selected for CABG stratum and subsequently, 378 patients were randomly assigned to revascularization arm within the CABG stratum. Our study cohort consisted of the 378 patients. Fifty-nine (16\%) patients received DAPT and 319 (84\%) patients received aspirin alone. Forty-one (70\%) patients remained on DAPT at 6 months. Baseline differences between patients who received DAPT and those who received aspirin monotherapy are outlined in Table 1. The presence of totally occluded lesions was more common in patients who received DAPT and a significant proximal left anterior descending artery lesion was more commonly present in patients who received aspirin monotherapy.

The outcomes are outlined in Table 2 and shown in Figure 1. No significant differences were observed in the 5 -year composite outcome of all-cause death, MI, or stroke in patients who received DAPT compared to those who received aspirin monotherapy: [13 (22.0\%) vs. 61 (19.1\%); adjusted hazard ratio (HR): $1.06 ; 95 \%$ confidence interval (CI): 0.56 to $2.00 ; \mathrm{P}=0.86]$. There was no significant difference in the need for subsequent CABG or PCI [4 (6.8\%) vs. 23 (7.2\%); HR: 1.21 ; $95 \%$ CI: 0.39 to 3.69 ;

Table 1 Baseline characteristics of patients who received DAPT and aspirin monotherapy

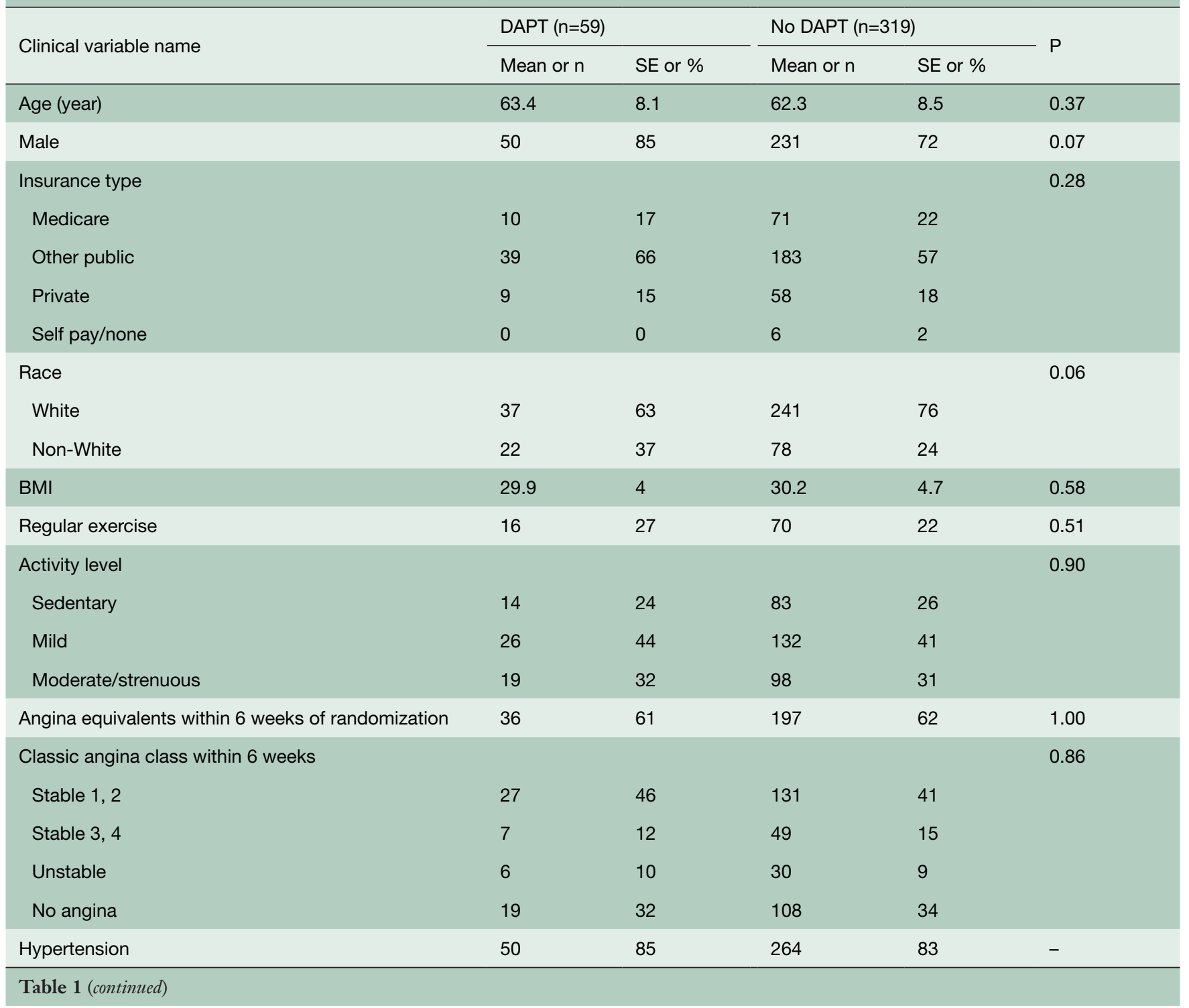




\begin{tabular}{|c|c|c|c|c|c|}
\hline \multirow{2}{*}{ Clinical variable name } & \multicolumn{2}{|c|}{ DAPT $(n=59)$} & \multicolumn{2}{|c|}{ No DAPT $(n=319)$} & \multirow{2}{*}{$\mathrm{P}$} \\
\hline & Mean or $n$ & SE or \% & Mean or $n$ & SE or $\%$ & \\
\hline Glycemic treatment arm & & & & & 0.24 \\
\hline Insulin sensitizing & 34 & 58 & 154 & 48 & \\
\hline Insulin provisional & 25 & 42 & 165 & 52 & \\
\hline Insulin use & 14 & 24 & 63 & 20 & 0.60 \\
\hline History of hypoglycemic episode & 8 & 14 & 55 & 17 & - \\
\hline Duration of diabetes (year) & 11.2 & 8.8 & 9.9 & 8 & 0.32 \\
\hline Waist circumference $(\mathrm{cm})$ & 105.4 & 12 & 104.6 & 11.9 & 0.68 \\
\hline Current tobacco use & & & & & 0.49 \\
\hline Current smoker & 5 & 8 & 38 & 12 & \\
\hline Former smoker & 35 & 59 & 163 & 51 & \\
\hline Never smoked & 19 & 32 & 117 & 37 & \\
\hline History of MI & 28 & 47 & 98 & 31 & 0.02 \\
\hline History of $\mathrm{CHF}$ requiring treatment & 2 & 3 & 16 & 5 & 1.00 \\
\hline LVEF $<50 \%$ & 9 & 16 & 58 & 18 & 0.77 \\
\hline Myocardial jeopardy score & 56.7 & 21.6 & 61.3 & 21.4 & 0.14 \\
\hline Prior stent & 4 & 7 & 18 & 6 & 0.76 \\
\hline Prior revascularization & 7 & 12 & 36 & 11 & 1.00 \\
\hline Stroke or transient ischemic attack & 5 & 8 & 29 & 9 & 1.00 \\
\hline Non-coronary arterial disease & 15 & 25 & 76 & 24 & 0.93 \\
\hline Ankle brachial index & 1.1 & 0.3 & 1 & 0.2 & 0.02 \\
\hline Creatinine & 1.1 & 0.3 & 1.1 & 0.3 & 0.74 \\
\hline Hemoglobin A1c & 7.5 & 1.8 & 7.7 & 1.7 & 0.55 \\
\hline Number of lesions $\geq 20 \%$ & 5.9 & 2.7 & 5.7 & 2.4 & 0.72 \\
\hline Lesions $\geq 50 \%$ stenosis & 3.6 & 2 & 3.7 & 1.7 & 0.81 \\
\hline Lesions $\geq 70 \%$ stenosis & 1.6 & 1.1 & 1.8 & 1.3 & 0.14 \\
\hline Proximal LAD $\geq 50 \%$ stenosis & 5 & 8 & 67 & 21 & 0.04 \\
\hline Totally occluded lesions & 45 & 76 & 191 & 60 & 0.02 \\
\hline Number of diseased regions ( $\geq 50 \%$ ) & 2.4 & 0.6 & 2.4 & 0.7 & 0.52 \\
\hline Region of enrollment & & & & & 0.05 \\
\hline USA & 17 & 29 & 138 & 43 & \\
\hline Non-USA & 42 & 71 & 181 & 57 & \\
\hline
\end{tabular}

Italic $\mathrm{P}$ values signify those that are statistically significant $(\mathrm{P}<0.05)$. CABG, coronary artery bypass grafting; $\mathrm{PCl}$, percutaneous coronary intervention; ASA, acetylsalicylic acid; DAPT, dual antiplatelet therapy. 
Table 2 Cox proportional hazard model for primary and secondary outcomes

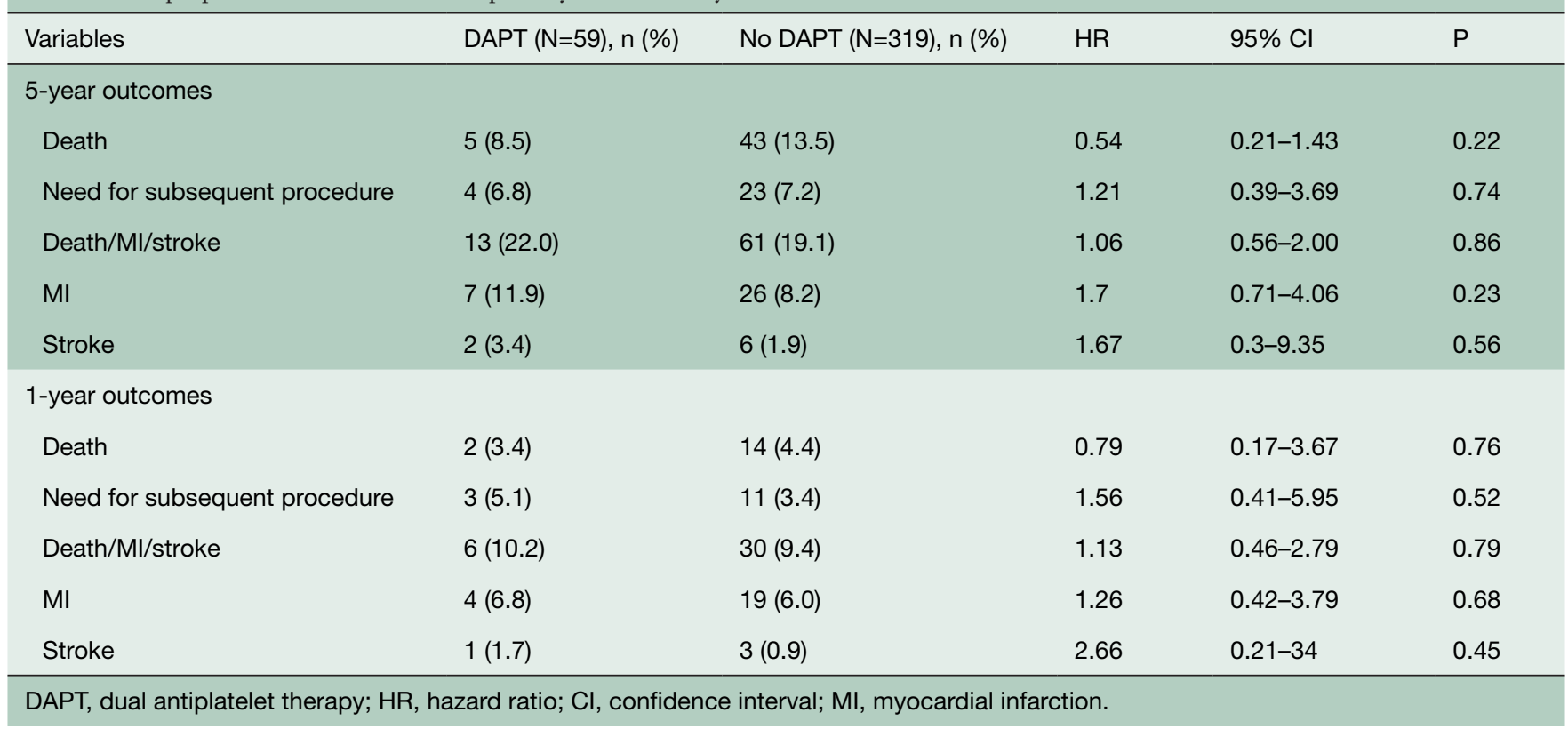

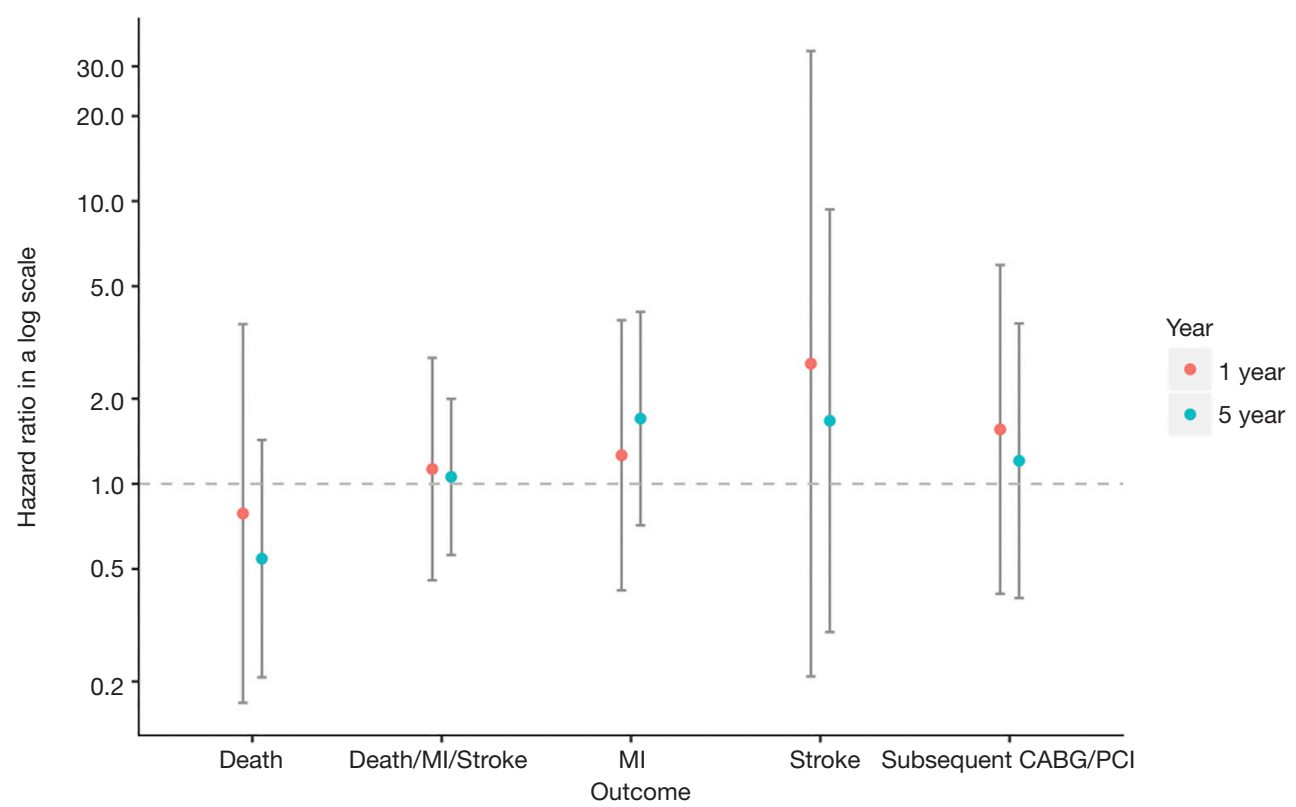

Figure 1 Hazard of 5-year MACCE associated with DAPT use following CABG. Shown above are the HRs and 95\% CI for adjusted hazard of 5-year outcomes associated with DAPT and aspirin monotherapy use. MACCE, major adverse cardiac and cerebrovascular events; DAPT, dual antiplatelet therapy; CABG, coronary artery bypass grafting; HR, hazard ratio; CI, confidence interval. 
$\mathrm{P}=0.74]$. There also was no significant difference at 1 year in the composite event [6 (10.2\%) vs. 30 (9.4\%); HR: 1.13; 95\% CI: 0.46 to $2.79 ; \mathrm{P}=0.79$ ] or need for subsequent CABG or PCI [3 (5.1\%) vs. 11 (3.4\%); HR: 1.56; 95\% CI: 0.41 to $5.95 ; \mathrm{P}=0.52]$.

\section{Discussion}

In this study, the rate of patients who were on DAPT following CABG in patients presenting with SIHD was low at $15.6 \%$. Cox proportional hazard regression demonstrated that the adjusted hazard for the primary endpoint of all-cause death, MI, or stroke was not statistically significantly different between patients on DAPT and aspirin monotherapy. In addition, the adjusted hazards of all secondary outcomes were not significantly different between the two cohorts. The findings support the hypothesis that DAPT does not provide therapeutic advantage over aspirin monotherapy in patients presenting with SIHD who subsequently undergo CABG.

The rate of DAPT use was significantly lower compared to that reported in FREEDOM (Future Revascularization Evaluation in patients with Diabetes mellitus: Optimal management of Multivessel disease) trial, which reported $68.4 \%$ of patients being on DAPT following CABG (10). This difference may be attributed to two factors: (I) in FREEDOM trial, financial compensation was provided to participants to offset the cost of DAPT, and (II) FREEDOM trial cohort consisted of $29 \%$ of patients who presented with ACS (10), for which guidelines recommend the use of DAPT following CABG (1-3). Using local institutional data, our group identified that the rate of DAPT use in a realworld setting was $29 \%$ in patients who presented with ACS and underwent CABG (11). In other clinical settings with a variable mix of ACS and SIHD cohorts, the rate of DAPT use following CABG ranges from $21 \%$ to $54 \%(12-15)$. Therefore, the lack of financial support to offset the cost of DAPT and non-ACS presentation in this cohort likely resulted in the relatively low rate of use.

The apparent lack of therapeutic benefit associated with DAPT use in preventing MACCE in SIHD patients is perhaps not surprising based on previous observations. In a secondary analysis of Arterial Revascularization Trial (ART), in which outcome associated with the use of bilateral internal thoracic arteries compared to single internal thoracic artery was evaluated, there was no significant difference in MACCE at 1 year between those who received DAPT and those on aspirin monotherapy following CABG (15). Of note, 34\% of the ART cohort presented with unstable angina. Similarly, in a secondary analysis of FREEDOM, there was no significant difference in MACCE associated with DAPT compared to aspirin monotherapy at 1 and 5 years following CABG for mixed indications (10). Subgroup analyses of this cohort by ACS and non-ACS indications also did not yield significant associations with MACCE. A subgroup analysis of CURE trial evaluating $16.5 \%$ of patients who underwent CABG only demonstrated a trend toward association between DAPT and reduction of MACCE: relative risk (RR) of 0.89 (95\% CI: 0.71-1.11) (6).

At this time, there is no strong evidence that demonstrates reduction of the incidences of MACCE by the use of DAPT in patients undergoing CABG regardless of the presenting symptoms. However, there are several single-center randomized controlled trials comparing aspirin monotherapy and DAPT with clopidogrel in patients who underwent CABG with various mixture of presenting symptoms $(7,8,16)$. None of the trials were powered to evaluate the effect of DAPT on MACCE, but have demonstrated superior vein graft patency in the DAPT cohort. By the use of surrogate outcomes, such studies suggest an evaluation of larger patient cohorts may ultimately allow for detection of a therapeutic benefit of DAPT, albeit with small benefit. Notably, there also exists a randomized controlled trial comparing aspirin monotherapy to DAPT in elective CABG patients that did not find difference in 1-year angiographic graft patency or degree of intimal hyperplasia by intravascular ultrasound (17).

Do patients with SIHD and ACS represent two distinct population following CABG? With regards to the native coronary arteries and surgical graft patency, perceived benefits of DAPT are the following: (I) stabilization of existing plaque, (II) preservation of surgical graft patency, and (III) continued protection of existing stents. An invivo examination of plaque morphologies of culprit lesions demonstrated high-risk features (smaller luminal area, greater plaque burden, presence of thin-cap fibroatheroma) to exist more commonly in patients with STEMI compared to NSTEMI, unstable angina, and stable CAD (18). Culprit plaque rupture and the presence of thin-cap fibroatheroma is more common in patients with ACS compared to SIHD (19). As CABG revascularizes flow-limited territories with new arterial and venous conduits, the exiting plaque burden and high-risk features of such plaques in the native coronary arteries of patients with ACS who underwent CABG may not manifest in a clinically significant difference compared 
to the SIHD population. Whether the presenting symptom of ACS or SIHD interacts with adverse event rate following CABG associated with DAPT still remains unclear, but our study may support non-use of DAPT in the SIHD population. The argument may be further supported by the presumption that patients with ACS are at higher risk of MACCE long term compared to those presenting with SIHD.

Although this study preceded the approval of such medications, of interest are the novel P2Y12 inhibitors or the 'higher-intensity' antiplatelet agents, such as prasugrel and ticagrelor. In a meta-analysis that included a subgroup analyses of patients in TRITON-TIMI-38 and PLATO trials who underwent CABG indicates that the 'higherintensity' antiplatelet agents, prasugrel and ticagrelor, are associated with reduction in the rate of MACCE compared to clopidogrel as the second antiplatelet agent (20). The observed benefits of prasugrel and ticagrelor in the CABG subgroup of TRITON-TIMI-38 and PLATO have not been validated in the absence of a trial designed to evaluate this in a CABG cohort.

There remains a need for more robust evidence that either supports or negates the proposed benefit of DAPT use following $\mathrm{CABG}$, and our study may support such trials to focus on patients presenting with ACS, not SIHD. The presenting symptoms should be delineated clearly in the inclusion criteria, as numerous trials have included patients with both ACS and SIHD to variable degree, while the guideline endorses DAPT use only in patients presenting with ACS undergoing CABG, although this is supported by limited evidence.

\section{Limitations}

The result of this study should be interpreted in the context of the following limitations. This is a non-randomized post-hoc analysis of randomized trial data, and therefore the two cohorts may have unbalanced characteristics not accounted for by the statistical adjustments. The use of DAPT declined over the follow-up period in the DAPT cohort patients, which may have impacted on the apparent lack of therapeutic benefit. The use of DAPT was relatively uncommon in this cohort, and the sample size may have underpowered the study, although all adjusted HR was in close proximity to the unity. The trial data did not delineate those patients undergoing off-pump CABG, a cohort in which the use of DAPT is strongly advocated (21), although data to support that practice is limited. Finally, safety endpoints (i.e., bleeding) could not be assessed, as the trial did not delineate this. Provided these limitations, the results should be considered hypothesis-generating.

\section{Conclusions}

The use of DAPT in patients with diabetes post-CABG in this cohort was low. Compared with aspirin monotherapy, no associated differences were observed in cardiovascular outcomes, suggesting that routine use of DAPT in diabetics with SIHD after CABG may not be clinically warranted.

\section{Acknowledgements}

This Manuscript was prepared using BARI2D Research Materials obtained from the NHLBI Biologic Specimen and Data Repository Information Coordinating Center and does not necessarily reflect the opinions or views of the BARI2D or the NHLBI. This study was funded by Yale Collaboration for Research Integrity and Transparency (CRIT).

\section{Footnote}

Conflicts of Interest: The authors have no conflicts of interest to declare.

\section{References}

1. Levine GN, Bates ER, Bittl JA, et al. 2016 ACC/ AHA Guideline Focused Update on Duration of Dual Antiplatelet Therapy in Patients With Coronary Artery Disease: A Report of the American College of Cardiology/ American Heart Association Task Force on Clinical Practice Guidelines: An Update of the 2011 ACCF/AHA/ SCAI Guideline for Percutaneous Coronary Intervention, 2011 ACCF/AHA Guideline for Coronary Artery Bypass Graft Surgery, 2012 ACC/AHA/ACP/AATS/PCNA/ SCAI/STS Guideline for the Diagnosis and Management of Patients With Stable Ischemic Heart Disease, 2013 ACCF/AHA Guideline for the Management of STElevation Myocardial Infarction, 2014 AHA/ACC Guideline for the Management of Patients With NonST-Elevation Acute Coronary Syndromes, and 2014 ACC/AHA Guideline on Perioperative Cardiovascular Evaluation and Management of Patients Undergoing Noncardiac Surgery. Circulation 2016;134:e123-55.

2. Tanguay JF, Bell AD, Ackman ML, et al. Focused 2012 
update of the Canadian Cardiovascular Society guidelines for the use of antiplatelet therapy. Can J Cardiol 2013;29:1334-45

3. Kolh P, Windecker S, Alfonso F, et al. 2014 ESC/EACTS Guidelines on myocardial revascularization: the Task Force on Myocardial Revascularization of the European Society of Cardiology (ESC) and the European Association for Cardio-Thoracic Surgery (EACTS). Developed with the special contribution of the European Association of Percutaneous Cardiovascular Interventions (EAPCI). Eur J Cardiothorac Surg 2014;46:517-92.

4. Yusuf S, Zhao F, Mehta SR, et al. Effects of clopidogrel in addition to aspirin in patients with acute coronary syndromes without ST-segment elevation. N Engl J Med 2001;345:494-502.

5. Wallentin L, Becker RC, Budaj A, et al. Ticagrelor versus clopidogrel in patients with acute coronary syndromes. $\mathrm{N}$ Engl J Med 2009;361:1045-57.

6. Fox KA, Mehta SR, Peters R, et al. Benefits and risks of the combination of clopidogrel and aspirin in patients undergoing surgical revascularization for non-ST-elevation acute coronary syndrome: the Clopidogrel in Unstable angina to prevent Recurrent ischemic Events (CURE) Trial. Circulation 2004;110:1202-8.

7. Mannacio VA, Di Tommaso L, Antignan A, et al. Aspirin plus clopidogrel for optimal platelet inhibition following off-pump coronary artery bypass surgery: results from the CRYSSA (prevention of Coronary arteRY bypaSS occlusion After off-pump procedures) randomised study. Heart 2012;98:1710-5.

8. Gao G, Zheng Z, Pi Y, et al. Aspirin plus clopidogrel therapy increases early venous graft patency after coronary artery bypass surgery a single-center, randomized, controlled trial. J Am Coll Cardiol 2010;56:1639-43.

9. Group BDS, Frye RL, August P, et al. A randomized trial of therapies for type 2 diabetes and coronary artery disease. N Engl J Med 2009;360:2503-15.

10. van Diepen S, Fuster V, Verma S, et al. Dual Antiplatelet Therapy Versus Aspirin Monotherapy in Diabetics With Multivessel Disease Undergoing CABG: FREEDOM Insights. J Am Coll Cardiol 2017;69:119-27.

11. Mori M, Shioda K, Yun JJ, et al. Pattern and predictors of dual antiplatelet use after coronary artery bypass graft surgery. J Thorac Cardiovasc Surg 2018;155:632-8.

12. Chan V, Kulik A, Bourke ME, et al. Clopidogrel is safe early after on- and off-pump coronary artery bypass surgery. J Card Surg 2007;22:493-7.
13. Sorensen R, Abildstrom SZ, Hansen PR, et al. Efficacy of post-operative clopidogrel treatment in patients revascularized with coronary artery bypass grafting after myocardial infarction. J Am Coll Cardiol 2011;57:1202-9.

14. Kim DH, Daskalakis C, Silvestry SC, et al. Aspirin and clopidogrel use in the early postoperative period following on-pump and off-pump coronary artery bypass grafting. J Thorac Cardiovasc Surg 2009;138:1377-84.

15. Benedetto U, Altman DG, Gerry S, et al. Impact of dual antiplatelet therapy after coronary artery bypass surgery on 1-year outcomes in the Arterial Revascularization Trial. Eur J Cardiothorac Surg 2017;52:456-61.

16. Gasparovic H, Petricevic M, Kopjar T, et al. Impact of dual antiplatelet therapy on outcomes among aspirinresistant patients following coronary artery bypass grafting. Am J Cardiol 2014;113:1660-7.

17. Kulik A, Le May MR, Voisine P, et al. Aspirin plus clopidogrel versus aspirin alone after coronary artery bypass grafting: the clopidogrel after surgery for coronary artery disease (CASCADE) Trial. Circulation 2010;122:2680-7.

18. Dong L, Mintz GS, Witzenbichler B, et al. Comparison of plaque characteristics in narrowings with ST-elevation myocardial infarction (STEMI), non-STEMI/unstable angina pectoris and stable coronary artery disease (from the ADAPT-DES IVUS Substudy). Am J Cardiol 2015;115:860-6.

19. Iannaccone M, Quadri G, Taha S, et al. Prevalence and predictors of culprit plaque rupture at OCT in patients with coronary artery disease: a meta-analysis. Eur Heart J Cardiovasc Imaging 2016;17:1128-37.

20. Verma S, Goodman SG, Mehta SR, et al. Should dual antiplatelet therapy be used in patients following coronary artery bypass surgery? A meta-analysis of randomized controlled trials. BMC Surg 2015;15:112.

21. Gurbuz AT, Zia AA, Vuran AC, et al. Postoperative clopidogrel improves mid-term outcome after off-pump coronary artery bypass graft surgery: a prospective study. Eur J Cardiothorac Surg 2006;29:190-5.

Cite this article as: Mori M, Shioda $\mathrm{K}$, Bin Mahmood SU, Mangi AA, Yun JJ, Geirsson A. Dual antiplatelet therapy versus aspirin monotherapy in diabetics with stable ischemic heart disease undergoing coronary artery bypass grafting. Ann Cardiothorac Surg 2018;7(5):628-635. doi: 10.21037/ acs.2018.08.01 